\title{
Optimal Planning of Web Application in IIS Application Pool Fengyu Han ${ }^{1, a^{*}}$ and Qiang $\mathrm{Fu}^{2, \mathrm{~b}}$ \\ ${ }^{1,2}$ Changchun University of Science and Technology, China Jilin Changchun 130022 atuhanfengyu@163.com, bfuqiangwork@163.com
}

Keywords: Application pool; Worker process; IIS; Web Garden.

\begin{abstract}
Until IIS6 the concept of application pool began to emerge. Its function is the management process. Because the application pool can isolate different applications in separate memory pool, which makes the application in the application pool will not be amiss to affect the application of other application pools. At the same time, putting a number of programs in different application pool will consume more memory and CPU. The objective of this article is to determine the optimal planning method to application in the application pool. This paper first introduced the concept of the application pool, the worker process and the web garden. And then, according to an example comparison to compare with three kinds of planning methods in the application pool. Finally, analysis of the Web program in three different ways of planning, that we can obtain the optimal planning which for different the web application.
\end{abstract}

\section{Application Pool [1, 2]}

WAS (Web Administration Service) provides one of the components is the application pool. Applications are isolated in different application pools to ensure that they don't affect each other, which adds to the reliability and robustness of applications. Each application pool usually manages a worker process. But when we use the Web Garden or some exception occurred can lead to an application pool to manage multiple process. What's more, a worker process can include multiple applications. Then the article explains the meaning of the worker process in detail and the way of Web Garden works.

W3wp.exe [3]. As we all know, W3wp.exe is a process associated with the application pool in IIS. Process's main function is to allocate system resources, process requests, and store data for application programs. One important thing to note is that, closing the web program doesn't cause the W3wp.exe to close. Worker process will be automatically recovered when the "idle timeout" is reached.

Web Garden [4]. Web Garden can create multiple worker processes in an application pool, meaning that allows multiple processes service to an application pool. As shown in Fig. 1. The request is passed along to the worker process when http.sys receives the request .Web Garden does a good job of using multiple worker process for distinct request. This means that Web Garden can do the following:

1) When a request to need a lot of time for processing, the Web Garden can still receive a new request.

2) To improve the stability of the application.

3) The request is processed according to the method in the worker process that can improve system performance. 


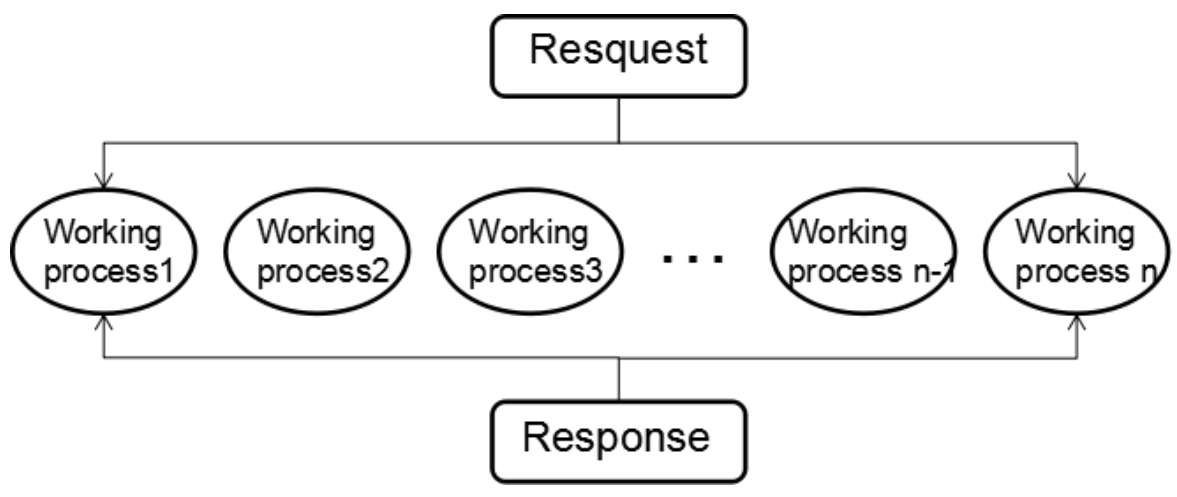

Figure 1.

\section{Three Planning Program [5,6]}

As a matter of fact, there doesn't have the application pool until IIS6. So before the IIS6, applications in a big "shared pool" what use low, intermediate and advanced isolation model to isolate the different applications. But now with the application pool, we can get three kind of planning for the storage of application in the pool.

1) An application for a pool;

2) Multiple applications in a pool;

3) Start the multiple processes in the pool (Web Garden).

Comparison of Three Methods for Planning. Using a concrete example to compare these three methods. We wrote a simple page that uses the language of HTML. And then to copy the web page three times. In the meantime, we will have four same web pages named htmll to html4 respectively. Putting html1 and html2 into two application pools which named demo1 and demo2. Next, putting html 3 and html4 into one pool named demo. At this point the contents of the task manager as shown in table 1 .

Table 1

\begin{tabular}{|l|l|l|l|l|l|l|}
\hline Name & PID & $\begin{array}{l}\text { Working } \\
\text { State }\end{array}$ & $\begin{array}{l}\text { Company } \\
\text { name }\end{array}$ & CPU & Memory & Description \\
\hline w3wp.exe & 980 & Running & demo1 & 00 & $12,068 \mathrm{~K}$ & IIS Worker Process \\
\hline w3wp.exe & 11280 & Running & demo2 & 00 & $11,940 \mathrm{~K}$ & IIS Worker Process \\
\hline w3wp.exe & 9944 & Running & demo & 00 & $20,192 \mathrm{~K}$ & IIS Worker Process \\
\hline
\end{tabular}

Sets the maximum number of the demo 1 and the demo 2 to three. After running html1 and html2 and seeing the task manager. Then we get the table 2. According to the figures shown in the table, each application pool has two processes at work [7]. Nevertheless the combined memory usage of two processes is more than ram occupation of a single process. 
Table 2

\begin{tabular}{|c|c|c|c|c|c|c|}
\hline Name & PID & $\begin{array}{c}\text { Working } \\
\text { State }\end{array}$ & $\begin{array}{c}\text { Company } \\
\text { name }\end{array}$ & CPU & Memory & Description \\
\hline w3wp.exe & 23512 & Running & demo1 & 00 & $3904 \mathrm{~K}$ & IIS Worker Process \\
\hline w3wp.exe & 24351 & Running & demo1 & 00 & $11656 \mathrm{~K}$ & IIS Worker Process \\
\hline w3wp.exe & 22264 & Running & demo2 & 00 & $3904 \mathrm{~K}$ & IIS Worker Process \\
\hline w3wp.exe & 37736 & Running & demo2 & 00 & $11844 \mathrm{~K}$ & IIS Worker Process \\
\hline
\end{tabular}

In addition, set the maximum number of demo to 6 . Run the program and review the task manager. The demo produced three worker processes. The application memory was higher due to the increased number of worker process.

Table 3

\begin{tabular}{|c|c|c|c|c|c|c|}
\hline Name & PID & $\begin{array}{c}\text { Working } \\
\text { State }\end{array}$ & $\begin{array}{c}\text { Company } \\
\text { name }\end{array}$ & CPU & Memory & Description \\
\hline w3wp.exe & 20913 & Running & demo & 00 & $3848 \mathrm{~K}$ & IIS Worker Process \\
\hline w3wp.exe & 15033 & Running & demo & 00 & $3856 \mathrm{~K}$ & IIS Worker Process \\
\hline w3wp.exe & 19214 & Running & demo & 0 & $14788 \mathrm{~K}$ & IIS Worker Process \\
\hline
\end{tabular}

Three examples are given to demonstrate that an application pool with multiple processes consume more memory and CPU time compared to the single process, so don't try to apply the Web Garden to web applications which consume large amounts of CPU and memory resources. A number of applications in an application pool that would reduce the occupation of resources, but it can also lead to more unstable and insecure.

\section{Working Condition of Three Kinds of Planning}

Ideally, launch site on IIS. In the case of the server's memory is $2 \mathrm{G}$. If each running application pool to occupy $60 \mathrm{M}$ memory and has only one website. So that up to 34 website online. But sometimes the application takes up more resources in that it is frequently accessed or talks to databases. So typically, better keep 30 to 40 applications pool on web Servers of IIS[8,9].

According to server hardware performance to choose the application of specific planning method in the application pool. If the hardware performance very well, the applications can be placed in a separate pool. But if you want to reduce the memory and CPU resources, and ensure the application's stability, security and usability. Must follow the following principles:

1) The application that requires a lot of interaction with the database should be placed in a separate application pool. For example, When student select courses in the educational administration system, that increase the frequency of access to the educational administration system, and system interaction with the database too much, so the system takes up the memory and CPU will be very high. When the CPU is already running at $100 \%$ for a long time that will cause the application pool to be recycled, and then affect other applications. So application of more 
resource consumption should be placed separately, and using Garden Web.

2) Put the program will not often use and occupy less memory (less than 30M) into one application pool.

3) To improve the user experience, a reasonable value should be chosen for maximum CPU utilization(percentages). When a worker process retrieves and accesses the database, possible CPU usage reaches $100 \%$, if before you enable CPU monitoring to close, the website will often lead to 503 errors while will reduce the user experience.

\section{Summary}

Use the application pool with extreme caution. Avoid Web Garden to be used in the application which consumes significant CPU resources, otherwise it will be frequently recovered because the application takes too much CPU resources. On one hand, we should have an accurate understanding of the server hardware. On the other hand, we should constantly monitor server's event logs, then find applications that take up too many resources [10], and re-planning.

\section{References}

[1] Willian R. Stonck: Administrator's Pocket Consultant (world publishing corporation, USA 2007), p.72

[2] Y Xia: Science \& Technology Information, (2010) No.17, p450. (In Chinese)

[3] Juliana Aldous Atkinson, Martin DelRe: Deploying microsoft internet information services (IIS) 6.0(Washington Microsoft Press, Redmond, 2003).

[4] Information on http://kb.cnblogs.com/page/122612/.

[5] Y.P Liu: China Computer \& Communication, (2009) No.10, p62. (In Chinese)

[6] M Gao, Z.Y Shi, M Huang: Journal of Changchun University of Technology (Natural Science Edition), Vol.25 (2004) No.3, p45. (In Chinese)

[7] Information on http://www.cnblogs.com/jankie/archive/2012/03/14/2396688.html.

[8] D.M Jiang: Continue Education Research, Vol.3 (2009) No.52, p61. (In Chinese)

[9]Tulloch: IIS6 Management Guide (Tsinghua University Press, Beijing, 2005), Vol.5 (2009) No.9, p2101. (In Chinese)

[10] Y.Z Wang: Computer Knowledge And Technology, Vol.3 (2008) No.8, p1689. (In Chinese) 